\title{
Conflicting shape percepts explained by perception cognition distinction
}

Paul Linton ${ }^{1}$

Morales et al. (1) show that a front-facing ellipse is more quickly identified amongst front-facing circles than rotated circles, leading them to conclude that vision has a dual character: a rotated circle is perceived both as a perspectival 2D ellipse and as a 3D circle. We have two concerns:

First, is this testing perceived shape? Morales et al. equate perspective with perceived 2D shape, but another way of thinking about perspective is $3 \mathrm{D}$ visual direction. The edge of a rotated 3D circle and a front-facing 2D ellipse have the same 3D visual direction even if the visual system has fully recovered the circle's 3D shape. This isn't just visually true, but physically true. If I ask someone without sight to point and trace out the direction of the edge of a rotated 3D circle, the path they will trace will be elliptical. Its 3D direction doesn't just look elliptical, it is elliptical. Similarly, if, when searching for a front-facing circle, a soccer ball distracts more than an American football, this doesn't necessarily imply that we must see the soccer ball as a $2 \mathrm{D}$ circle, only that the edge of a $2 \mathrm{D}$ circle and a 3D soccer ball share a common 3D visual direction. Just as there's no conflict between physical direction and physical shape, there's no conflict between 3D visual direction and 3D visual shape, although participants may be distracted by one when searching for the other.

Second, is this a failure of depth constancy? We argue that 3D shape isn't fully recovered for Morales et al.'s rotated 3D circles, which are perceived as closer to a 2D ellipse. First, pictures and movies of 3D objects (Experiments 1-7) are perceived as flatter than the physical objects they represent, either because pictures are cue conflict stimuli (2-3) or because pictorial cues are merely cognitive in nature (4-5). Second, whilst Morales et al.'s results persist in binocular viewing of real objects (Experiments 8-9), Johnston (6) found that perceived 3D shape from binocular disparity

\footnotetext{
${ }^{1}$ Centre for Applied Vision Research, City, University of London paul@linton.vision / https://linton.vision
} 
is flattened at distances greater than $80 \mathrm{~cm}$, so we would expect Morales et al.'s 3D rotated circles to be flattened at their $2.5 \mathrm{~m}$ viewing distance. It's true Morales et al.'s participants were able to identify their stimuli as representing a 3D circle, but we suggest that this identification of 3D shape is merely cognitive (4). For instance, whilst someone viewing a car and a photograph of a car may be able to identify the same object, they don't literally perceive the same 3D shape. Instead, to try and disentangle Morales et al.'s account from a failure of depth constancy, we should consider the case where an ellipse is compressed in the y-axis when viewed monocularly, but elongated when viewed binocularly (Figure 1). According to Morales et al., the monocular retinal image should compete with the binocular percept for our attention, but we find no evidence of this, and would expect reaction times to reflect this fact if participants were asked to judge whether the ellipse was elongated or compressed. 


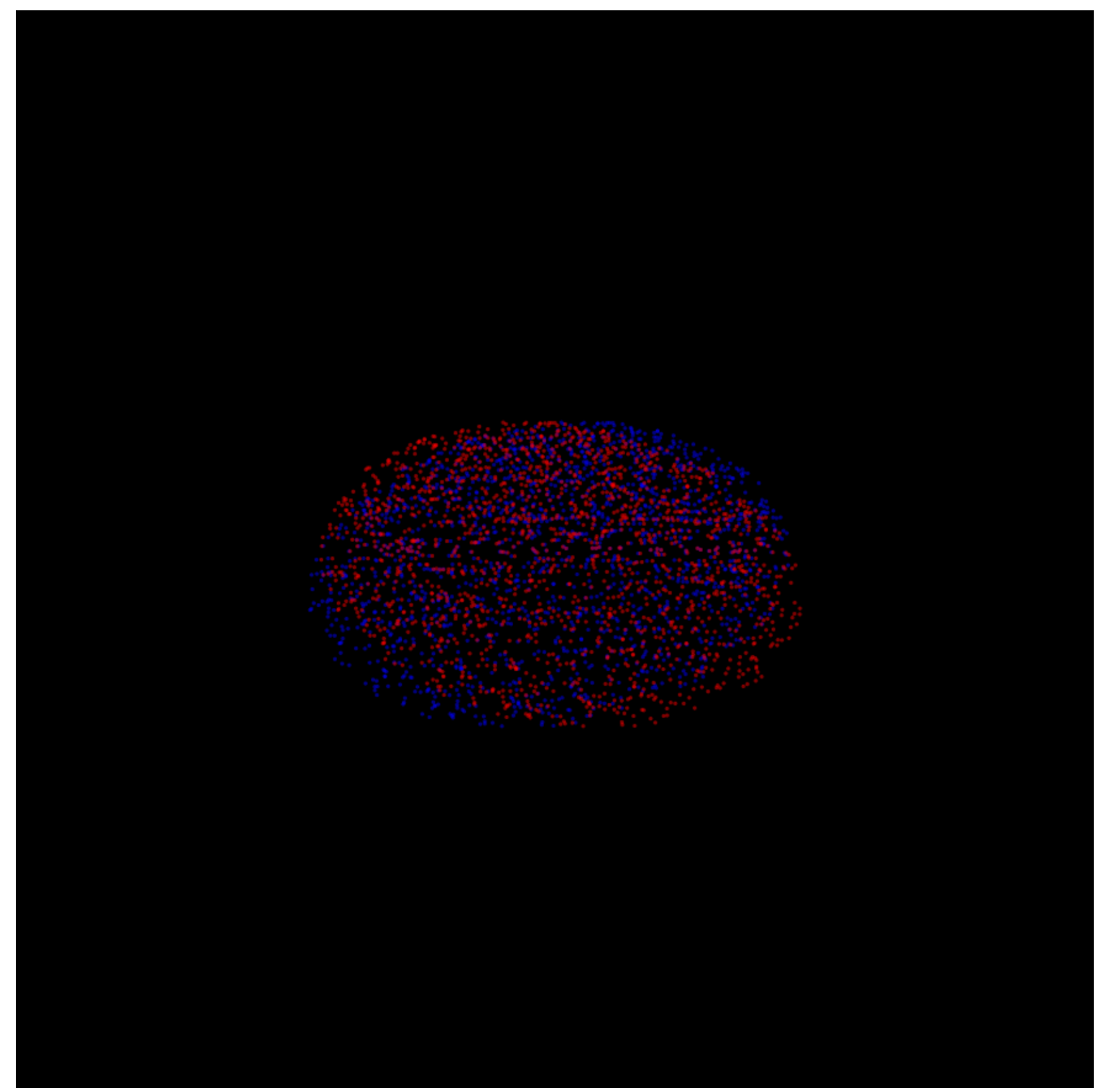

Figure 1. Slanted ellipse defined by binocular disparity. To be viewed with red-blue 3D glasses. 


\section{References}

1. J. Morales, A. Bax, C. Firestone, Sustained representation of perspectival shape. PNAS 117, 26, 14873-82 (2020).

2. J. M. Hillis, M. O. Ernst, M. S. Banks, M. S. Landy, Combining Sensory Information: Mandatory Fusion Within, but Not Between, Senses. Science 298, 5598, 1627-30 (2002).

3. D. C. Knill, Learning Bayesian priors for depth perception. J. Vis. 7, 8, 13 (2007).

4. P. Linton. The Perception and Cognition of Visual Space (Palgrave Macmillan, 2017).

5. O. Sacks, Stereo Sue: Why two eyes are better than one. New Yorker 82, 18, 64 (2006).

6. E. B. Johnston, Systematic distortions of shape from stereopsis. Vision Res. 31, 7, 1351-60 (1991). 